\title{
Sharp Bounds of the Hermitian Toeplitz Determinants for Certain Close-to-Star Functions
}

\section{Adam Lecko $^{1}$ (D) Barbara Śmiarowska ${ }^{1}$}

Received: 7 December 2021 / Revised: 18 January 2022 / Accepted: 31 January 2022 /

Published online: 19 February 2022

(c) The Author(s) 2022

\section{Abstract}

The sharp lower and upper bounds of the Hermitian Toeplitz determinants of the second and third order for certain close-to-star functions are computed.

Keywords Hermitian Toeplitz determinant · Univalent function · Close-to-star function · Close-to-convex functgion · Carathéodory class

Mathematics Subject Classification $30 \mathrm{C} 50 \cdot 30 \mathrm{C} 45$

\section{Introduction}

Let $\mathbb{D}:=\{z \in \mathbb{C}:|z|<1\}$ and $\overline{\mathbb{D}}:=\{z \in \mathbb{C}:|z| \leq 1\}$. Given $r>0$, let $\mathbb{T}_{r}:=\{z \in \mathbb{C}:|z|=r\}$ and $\mathbb{T}:=\mathbb{T}_{1}$. Let $\mathcal{H}$ denote the class of all analytic functions in $\mathbb{D}$ and $\mathcal{A}$ be its subclass of all functions $f$ normalized by $f(0)=0$ and $f^{\prime}(0)=1$, i.e., of the form

$$
f(z)=\sum_{n=1}^{\infty} a_{n} z^{n}, \quad a_{1}:=1, z \in \mathbb{D} .
$$

Let $\mathcal{S}$ be the subclass of $\mathcal{A}$ of univalent functions.

Communicated by Ali Abkar.

These authors contributed equally to this work.

$\triangle$ Adam Lecko

alecko@matman.uwm.edu.pl

Barbara Śmiarowska

b.smiarowska@matman.uwm.edu.pl

1 Department of Complex Analysis, Faculty of Mathematics and Computer Science, University of Warmia and Mazury in Olsztyn, ul. Słoneczna 54, Olsztyn 10-710, Poland 
Given $q, n \in \mathbb{N}$, define the matrix $T_{q, n}(f)$ of $f \in \mathcal{A}$ of the form (1.1) by

$$
T_{q, n}(f):=\left[\begin{array}{cccc}
a_{n} & a_{n+1} & \ldots & a_{n+q-1} \\
\bar{a}_{n+1} & a_{n} & \ldots & a_{n+q-2} \\
\vdots & \vdots & \vdots & \vdots \\
\bar{a}_{n+q-1} & \bar{a}_{n+q-2} & \ldots & a_{n}
\end{array}\right]
$$

where $\bar{a}_{k}:=\overline{a_{k}}$. When $a_{n}$ is a real number, $T_{q, n}(f)$ is the Hermitian Toeplitz matrix. In particular, any matrix $T_{q, 1}(f)$ so is.

In recent years, many authors studied the estimation of determinants whose entries are coefficients of functions in the class $\mathcal{A}$ or its subclasses. Hankel matrices, i.e., square matrices which have constant entries along the reverse diagonal and the generalized Zalcman functional $J_{m, n}(f):=a_{m+n-1}-a_{m} a_{n}, m, n \in \mathbb{N}$, are of particular interest (see, e.g., [5-7, 12, 14, 16-19, 23]. The determinants of symmetric Toeplitz matrices, the study of which was initiated in [1], are another example of such interest.

In $[8,10,13]$, the study to estimate the determinants $T_{q, n}(f)$ whose entries are coefficients of functions in subclasses of $\mathcal{A}$ was initiated. As it is well known, Hermitian Toeplitz matrices play an important role in functional analysis, applied mathematics and in technical sciences. Further results in this direction were obtained in [15].

In this paper, we continue this research by computing the sharp upper and lower bounds of determinants $T_{2,1}(f)$ and $T_{3,1}(f)$ over subclasses of close-to-star functions.

It can be observed that for each $\theta \in \mathbb{R}$, det $T_{q, 1}(f)=\operatorname{det} T_{q, 1}\left(f_{\theta}\right)$, where $f_{\theta}(z)$ $:=\mathrm{e}^{-\mathrm{i} \theta} f\left(\mathrm{e}^{\mathrm{i} \theta} z\right), z \in \mathbb{D}$, i.e., det $T_{q, 1}(f)$ is rotation invariant.

Theorem 1.1 [8] Let $\mathcal{F}$ be a subclass of $\mathcal{A}$ such that $\left\{f \in \mathcal{F}: a_{2}=0\right\} \neq \varnothing$ and $A_{2}(\mathcal{F}):=\max \left\{\left|a_{2}\right|: f \in \mathcal{F}\right\}$ exists. Then,

$$
1-A_{2}^{2}(\mathcal{F}) \leq \operatorname{det} T_{2,1}(f) \leq 1
$$

Both inequalities are sharp.

Let $\mathcal{S}^{*}$ denote the subclass of $\mathcal{S}$ of starlike functions, i.e., $f \in \mathcal{S}^{*}$ if $f \in \mathcal{A}$ and

$$
\operatorname{Re} \frac{z f^{\prime}(z)}{f(z)}>0, \quad z \in \mathbb{D} .
$$

A function $f \in \mathcal{A}$ is called close-to-star if there exist $g \in \mathcal{S}^{*}$ and $\beta \in \mathbb{R}$ such that

$$
\operatorname{Re} \frac{\mathrm{e}^{\mathrm{i} \beta} f(z)}{g(z)}>0, \quad z \in \mathbb{D}
$$

Recall that the class $\mathcal{C S T}$ of all close-to-star functions introduced by Reade [24] bear the same relation to the class of close-to-convex functions as the class of starlike functions bear to the class of convex functions ([24, p. 61])). This relationship is called 
Alexander type. Namely, $f \in \mathcal{C S} \mathcal{T}$ if and only if a function

$$
F(z):=\int_{0}^{z} \frac{f(t)}{t} \mathrm{~d} t, \quad z \in \mathbb{D}
$$

is close-to-convex ([11], [9, Vol. II, p. 3]). The class of close-to-convex functions was introduced by Kaplan [11], where the following geometrical interpretation was shown: $f \in \mathcal{A}$ is close-to-convex if and only if there are no sections of the curve $f\left(\mathbb{T}_{r}\right)$, for every $r \in(0,1)$, in which the tangent vector turns backward through an angle not less then $\pi$ (cf. [9, Vol. II, p. 4]). An analogous geometrical interpretation for close-to-star functions was shown by Reade: $f \in \mathcal{A}$ is close-to-star if and only if there are no sections of the curve $f\left(\mathbb{T}_{r}\right)$, for every $r \in(0,1)$, in which the radius vector to the curve $f\left(\mathbb{T}_{r}\right)$ turns backward through an angle not less than $\pi$ ( [24, p. 61]). Recall also that Lewandowski [20] proved that the class of close-to-convex functions is identical to the class of linearly accessible functions introduced by Biernacki [2].

The class of close-to-star functions and their subclasses were studied by various authors (e.g., MacGregor [21], Sakaguchi [26], Causey and Merkes [4]; for further references, see [9, Vol. II, pp. 97-104]).

Given $g \in \mathcal{S}^{*}$ and $\beta \in \mathbb{R}$, let $\mathcal{C S T} \mathcal{T}_{\beta}(g)$ are the subclass of $\mathcal{C S T}$ of all $f$ satisfying (1.2). The four classes $\mathcal{C S} \mathcal{T}_{0}\left(g_{i}\right), i=1, \ldots, 4$ where

$$
g_{1}(z):=\frac{z}{1-z^{2}}, \quad g_{2}(z):=\frac{z}{(1-z)^{2}}, \quad g_{3}(z):=\frac{z}{1+z+z^{2}}
$$

and

$$
g_{4}(z):=\frac{z}{1-z}, \quad z \in \mathbb{D}
$$

are particularly interesting and were separately studied by various authors. In [10], the sharp bounds of the second- and third-order Hermitian Toeplitz determinants were computed for the classes $\mathcal{C} \mathcal{S} \mathcal{T}_{0}\left(g_{1}\right)$ and $\mathcal{C S} \mathcal{T}_{0}\left(g_{2}\right)$. In this paper, we will estimate the second- and third-order Hermitian Toeplitz determinants for the other two classes, i.e., for $\mathcal{C S T}_{0}\left(g_{3}\right)=: \mathcal{F}_{1}$ and $\mathcal{C S T}_{0}\left(g_{4}\right)=: \mathcal{F}_{2}$, in which elements $f$ in view of (1.2) satisfy the condition

$$
\operatorname{Re}\left\{\left(1+z+z^{2}\right) \frac{f(z)}{z}\right\}>0, \quad z \in \mathbb{D}
$$

and

$$
\operatorname{Re}\left\{(1-z) \frac{f(z)}{z}\right\}>0, \quad z \in \mathbb{D}
$$

respectively. Let us add that every function $f$ in $\mathcal{F}_{1}$ analytic in the closed disk $\overline{\mathbb{D}}$ is starlike in one direction as defined by Robertson [25] (see also [9, Vol. I, pp. 207-208] 
and [9, Vol. I, p. 210, Theorem 27] with $\mu=0$ and $v=2 \pi / 3)$ ), i.e., intersection of $f(\mathbb{D})$ with the real axis is a line segment covered by $f$ univalently. Note that

$$
\operatorname{det} T_{3,1}(f)=\left|\begin{array}{ccc}
1 & a_{2} & a_{3} \\
\bar{a}_{2} & 1 & a_{2} \\
\bar{a}_{3} & \bar{a}_{2} & 1
\end{array}\right|=2 \operatorname{Re}\left(a_{2}^{2} \bar{a}_{3}\right)-2\left|a_{2}\right|^{2}-\left|a_{3}\right|^{2}+1
$$

Let $\mathcal{P}$ be the class of all $p \in \mathcal{H}$ of the form

$$
p(z)=1+\sum_{n=1}^{\infty} c_{n} z^{n}, \quad z \in \mathbb{D}
$$

having a positive real part in $\mathbb{D}$.

In the proof of the main result, we will use the following lemma, which contains the well-known formula for $c_{2}$ ([3], [22, p. 166]) and further remarks in [7]). In fact, the formulas below follow also from the Carathéodory-Toeplitz theorem.

Lemma 1.2 If $p \in \mathcal{P}$ is of the form (1.6), then

$$
\left|c_{n}\right| \leq 2, \quad n \in \mathbb{N}
$$

Moreover,

$$
c_{1}=2 \zeta_{1}
$$

and

$$
c_{2}=2 \zeta_{1}^{2}+2\left(1-\left|\zeta_{1}\right|^{2}\right) \zeta_{2}
$$

for some $\zeta_{i} \in \overline{\mathbb{D}}, i \in\{1,2\}$.

For $\zeta_{1} \in \mathbb{T}$, there is a unique function $p \in \mathcal{P}$ with $c_{1}$ as in (1.8), namely,

$$
p(z)=\frac{1+\zeta_{1} z}{1-\zeta_{1} z}, \quad z \in \mathbb{D}
$$

For $\zeta_{1} \in \mathbb{D}$ and $\zeta_{2} \in \mathbb{T}$, there is a unique function $p \in \mathcal{P}$ with $c_{1}$ and $c_{2}$ as in (1.8) and (1.9), namely,

$$
p(z)=\frac{1+\left(\bar{\zeta}_{1} \zeta_{2}+\zeta_{1}\right) z+\zeta_{2} z^{2}}{1+\left(\bar{\zeta}_{1} \zeta_{2}-\zeta_{1}\right) z-\zeta_{2} z^{2}}, \quad z \in \mathbb{D}
$$




\section{The Class $\mathcal{F}_{1}$}

Let $f \in \mathcal{F}_{1}$ be the form (1.1). Then by (1.3), there exists $p \in \mathcal{P}$ of the form (1.6) such that

$$
\left(1+z+z^{2}\right) \frac{f(z)}{z}=p(z), \quad z \in \mathbb{D} .
$$

Substituting the series (1.1) and (1.6) into (2.1) by equating the coefficients, we get

$$
a_{2}=-1+c_{1} \text { and } a_{3}=-c_{1}+c_{2}
$$

By (1.7), it follows that $A_{2}\left(\mathcal{F}_{1}\right)=3$ for the function $f \in \mathcal{A}$ defined by

$$
f(z)=\frac{z(1-z)}{\left(1+z+z^{2}\right)(1+z)}, \quad z \in \mathbb{D},
$$

which is extremal. Observe also that $a_{2}=0$ for the function $f \in \mathcal{F}_{1}$ defined by

$$
f(z)=\frac{z}{\left(1+z+z^{2}\right)(1-z)}, \quad z \in \mathbb{D} .
$$

Therefore by Theorem 1.1, we have

Theorem 2.1 If $f \in \mathcal{F}_{1}$, then

$$
-8 \leq \operatorname{det} T_{2,1}(f) \leq 1 \text {. }
$$

Both inequalities are sharp.

Now, we will compute the bounds of $\operatorname{det} T_{3,1}(f)$ in the class $\mathcal{F}_{1}$.

Theorem 2.2 If $f \in \mathcal{F}_{1}$, then

$$
\operatorname{det} T_{3,1}(f) \leq 39 \text {. }
$$

The inequality is sharp.

Proof By (2.2) and (1.7), we see that $\left|a_{2}\right| \leq 3$ and $\left|a_{3}\right| \leq 4$. Since $\operatorname{Re}\left(a_{2}^{2} \bar{a}_{3}\right)$ $\leq\left|a_{2}\right|^{2}\left|a_{3}\right|$ from (1.5) we get

$$
\operatorname{det} T_{3,1}(f) \leq F\left(\left|a_{2}\right|,\left|a_{3}\right|\right)
$$

where

$$
F(x, y):=2 x^{2} y-2 x^{2}-y^{2}+1, \quad(x, y) \in[0,3] \times[0,4] .
$$


Observe that the point $(1,1)$ is the unique solution $(0,3) \times(0,4)$ of the system of equations

$$
\begin{aligned}
& \frac{\partial F}{\partial x}=4 x(y-1)=0, \\
& \frac{\partial F}{\partial y}=2\left(x^{2}-y\right)=0 .
\end{aligned}
$$

However,

$$
\frac{\partial^{2} F}{\partial x^{2}}(1,1) \frac{\partial^{2} F}{\partial y^{2}}(1,1)-\left(\frac{\partial F}{\partial x \partial y}(1,1)\right)^{2}=-16<0
$$

so $(1,1)$ is a saddle point of $F$.

We now consider $F$ on the boundary of $[0,3] \times[0,4]$.

(1) On the side $x=0$,

$$
F(0, y)=1-y^{2} \leq 1, \quad 0 \leq y \leq 4
$$

(2) On the side $x=3$,

$$
F(3, y)=-17+18 y-y^{2} \leq F(3,4)=39, \quad 0 \leq y \leq 4 .
$$

(3) On the side $y=0$,

$$
F(x, 0)=1-2 x^{2} \leq 1, \quad 0 \leq x \leq 3
$$

(4) On the side $y=4$

$$
F(x, 4)=-15+6 x^{2} \leq 39, \quad 0 \leq x \leq 3
$$

Therefore, the inequality $F(x, y) \leq 39$ holds for all $(x, y) \in[0,3] \times[0,4]$, which in view of (2.5) shows (2.4).

For the function (2.3), $a_{2}=-3$ and $a_{3}=4$, which makes equality in (2.4).

Theorem 2.3 If $f \in \mathcal{F}_{1}$, then

$$
\begin{aligned}
\operatorname{det} T_{3,1}(f) & \geq \frac{1}{8}\left[(6 \sqrt{2}-15)(4+2 \sqrt{2})^{\frac{2}{3}}+(3 \sqrt{2}-18)(4+2 \sqrt{2})^{\frac{1}{3}}-36\right] \\
& =-10.693535 \ldots
\end{aligned}
$$

The inequality is sharp.

Proof Substituting (1.8) and (1.9) into (2.2), we get

$$
a_{2}=-1+2 \zeta_{1}, \quad a_{3}=-2 \zeta_{1}+2 \zeta_{1}^{2}+2\left(1-\left|\zeta_{1}\right|^{2}\right) \zeta_{2}
$$


with $\zeta_{i} \in \overline{\mathbb{D}}, i=1,2$. Therefore from (1.5), we get

$$
\operatorname{det} T_{3,1}(f)=\Psi_{1}+\Psi_{2}
$$

where

$$
\Psi_{1}:=-1+4\left|\zeta_{1}\right|^{2}+12\left|\zeta_{1}\right|^{4}-4\left(1-\left|\zeta_{1}\right|^{2}\right)^{2}\left|\zeta_{2}\right|^{2}
$$

and

$$
\begin{aligned}
\Psi_{2}:= & 4 \operatorname{Re} \zeta_{1}+4 \operatorname{Re}\left(\zeta_{1}^{2}\right)-24\left|\zeta_{1}\right|^{2} \operatorname{Re} \zeta_{1}-8\left(1-\left|\zeta_{1}\right|^{2}\right) \operatorname{Re}\left(\zeta_{1} \bar{\zeta}_{2}\right) \\
& +4\left(1-\left|\zeta_{1}\right|^{2}\right) \operatorname{Re} \zeta_{2}+8\left(1-\left|\zeta_{1}\right|^{2}\right) \operatorname{Re}\left(\zeta_{1}^{2} \bar{\zeta}_{2}\right)
\end{aligned}
$$

A. Let $\zeta_{1} \zeta_{2} \neq 0$. Thus $\zeta_{1}=r \mathrm{e}^{\mathrm{i} \theta}$ and $\zeta_{2}=s \mathrm{e}^{\mathrm{i} \psi}$ with $r, s \in(0,1]$ and $\theta, \psi \in[0,2 \pi)$. Then,

$$
\Psi_{2}=\Psi_{3}+\Psi_{4}
$$

where

$$
\begin{aligned}
\Psi_{3} & :=4 r \cos \theta+4 r^{2} \cos 2 \theta-24 r^{3} \cos \theta \\
& =-4 r^{2}+4 r \cos \theta-24 r^{3} \cos \theta+8 r^{2} \cos ^{2} \theta
\end{aligned}
$$

and

$$
\begin{aligned}
\Psi_{4} & :=-8 r s\left(1-r^{2}\right) \cos (\theta-\psi)+4 s\left(1-r^{2}\right) \cos \psi+8 r^{2} s\left(1-r^{2}\right) \cos (2 \theta-\psi) \\
& =4 s\left(1-r^{2}\right) \sqrt{\kappa_{1}^{2}+\kappa_{2}^{2}} \sin (\psi+\alpha),
\end{aligned}
$$

where $\alpha \in \mathbb{R}$ is the quantity satisfying

$$
\cos \alpha=\frac{\kappa_{1}}{\sqrt{\kappa_{1}^{2}+\kappa_{2}^{2}}}, \quad \sin \alpha=\frac{\kappa_{2}}{\sqrt{\kappa_{1}^{2}+\kappa_{2}^{2}}}
$$

with

$$
\kappa_{1}:=-2 r \sin \theta+2 r^{2} \sin 2 \theta, \quad \kappa_{2}:=1-2 r \cos \theta+2 r^{2} \cos 2 \theta .
$$

Since $\sin (\psi+\alpha) \geq-1$ and $s \leq 1$, we have

$$
\begin{aligned}
\Psi_{4} & \geq-4\left(1-r^{2}\right) \sqrt{\kappa_{1}^{2}+\kappa_{2}^{2}} \\
& =-4\left(1-r^{2}\right) \sqrt{1+4 r^{4}-4 r \cos \theta-8 r^{3} \cos \theta+8 r^{2} \cos ^{2} \theta}
\end{aligned}
$$


Therefore, from (2.9) to (2.11), we get

$$
\begin{aligned}
\Psi_{2}= & \Psi_{3}+\Psi_{4} \geq 4 r \cos \theta+4 r^{2} \cos 2 \theta-24 r^{3} \cos \theta \\
& -4\left(1-r^{2}\right) \sqrt{1+4 r^{4}-4 r \cos \theta-8 r^{3} \cos \theta+8 r^{2} \cos ^{2} \theta} .
\end{aligned}
$$

Taking into account that $\left|\zeta_{2}\right| \leq 1$ from (2.8), we have

$$
\Psi_{1} \geq-5+12 r^{2}+8 r^{4}
$$

Thus from (2.7), (2.14) and (2.15), it follows that

$$
\operatorname{det} T_{3,1}(f) \geq G(r, \cos \theta), \quad r \in(0,1], \theta \in[0,2 \pi),
$$

where

$$
G(t, x):=g_{1}(t, x)-4\left(1-t^{2}\right) \sqrt{g_{2}(t, x)}
$$

with

$$
g_{1}(t, x):=-5+8 t^{2}+8 t^{4}+4 t x-24 t^{3} x+8 t^{2} x^{2}
$$

and

$$
g_{2}(t, x):=1+4 t^{4}-4 t x-8 t^{3} x+8 t^{2} x^{2}
$$

for $t \in[0,1]$ and $x \in[-1,1]$.

Let $\Omega:=[0,1] \times[-1,1]$ and

$$
\begin{aligned}
\Theta: & =\frac{1}{8}\left[(6 \sqrt{2}-15)(4+2 \sqrt{2})^{\frac{2}{3}}+(3 \sqrt{2}-18)(4+2 \sqrt{2})^{\frac{1}{3}}-36\right] \\
& =-10.693535 \ldots
\end{aligned}
$$

Now, we will show that

$$
\min \{G(t, x):(t, x) \in \Omega\}=\Theta .
$$

A1. We now deal with the critical points of $G$ in the interior of $\Omega$, i.e., in $(0,1) \times$ $(-1,1)$. Note that $g_{2}(t, x) \geq 0$. Moreover, $g_{2}(t, x)=0$ holds for $t=\sqrt{2} / 2$ and $x=\sqrt{2} / 2$. A1.1. When $t=\sqrt{2} / 2$ and $x=\sqrt{2} / 2$, from (2.8), (2.10) and (2.11), we have

$$
\Psi_{1}=4-\left|\zeta_{2}\right|^{2}, \quad \Psi_{3}=-4, \quad \Psi_{4}=0
$$

which yields

$$
\operatorname{det} T_{3,1}(f) \geq-1 \text {. }
$$


A1.2. Suppose now that $g_{2}(t, x)>0$. Differentiating $G$ with respect to $x$ yields

$$
\frac{\partial G}{\partial x}(t, x)=\frac{\partial g_{1}}{\partial x}(t, x)-2\left(1-t^{2}\right)\left(g_{2}(t, x)\right)^{-1 / 2} \frac{\partial g_{2}}{\partial x}(t, x)=0 .
$$

A1.2.a. Assume first that $\partial g_{1} / \partial x=0$. Then from (2.18), it follows that $\partial g_{2} / \partial x=0$, which is possible only for $t=\sqrt{2} / 2$ and $x=\sqrt{2} / 2$. Further argumentation is as in A1.1. A1.2.b Assume now that $\partial g_{1} / \partial x \neq 0$. Then we can write the equation (2.18) as

$$
g_{2}(t, x)^{1 / 2}=\frac{2\left(1-t^{2}\right) \frac{\partial g_{2}}{\partial x}(t, x)}{\frac{\partial g_{1}}{\partial x}(t, x)}=\frac{2\left(1-t^{2}\right)\left(-1-2 t^{2}+4 t x\right)}{1-6 t^{2}+4 t x}
$$

or equivalently by substituting (2.17) as

$$
\begin{aligned}
\Phi=\Phi(t, x):= & 128 t^{8}-416 t^{7} x+672 t^{6} x^{2}-512 t^{5} x^{3}+128 t^{4} x^{4}-32 t^{6} \\
& -112 t^{5} x+160 t^{4} x^{2}+52 t^{4}-8 t^{3} x-72 t^{2} x^{2} \\
& -20 t^{2}+36 t x-3=0 .
\end{aligned}
$$

Furthermore, note that by (2.19),

$$
0<\frac{\left(g_{2}(t, x)\right)^{1 / 2}}{2\left(1-t^{2}\right)}=\frac{-1-2 t^{2}+4 t x}{1-6 t^{2}+4 t x}
$$

and this inequality holds for

$$
0<t<\frac{\sqrt{2}}{2} \text { and } \frac{2 t^{2}+1}{4 t}<x<\frac{6 t^{2}-1}{4 t}
$$

or

$$
\frac{\sqrt{2}}{2}<t<1 \text { and }\left(x<\frac{2 t^{2}+1}{4 t} \text { or } x>\frac{6 t^{2}-1}{4 t}\right) .
$$

Differentiating $G$ with respect to $t$ and using (2.19) yield

$$
\begin{aligned}
\frac{\partial G}{\partial t}(t, x) & =\frac{\partial g_{1}}{\partial t}(t, x)+8 t\left(g_{2}(t, x)\right)^{1 / 2}-2\left(1-t^{2}\right)\left(g_{2}(t, x)\right)^{-1 / 2} \frac{\partial g_{2}}{\partial t}(t, x) \\
& =-\frac{64 t^{2}}{\left(1-6 t^{2}+4 t x\right)\left(-1-2 t^{2}+4 t x\right)} H(t, x),
\end{aligned}
$$

where for $(t, x) \in(0,1) \times(-1,1)$,

$$
H(t, x):=(t-x)\left(-1-8 t^{2}+4 t^{4}+10 t x+4 t^{3} x-8 t^{2} x^{2}\right) .
$$


Therefore, each critical point of $G$ satisfies

$$
t-x=0
$$

or

$$
-1-8 t^{2}+4 t^{4}+10 t x+4 t^{3} x-8 t^{2} x^{2}=0 .
$$

I. Assume that (2.22) holds. Then, $x=x(t)=t$. Thus by (2.20), we see that

$$
\Phi(t, t)=\left(-3+4 t^{2}\right)\left(-1+2 t^{2}\right)^{2}=0
$$

occurs only when $t=\hat{t}_{i}, i=1,2$, where $\hat{t}_{1}:=\sqrt{3} / 2$ and $\hat{t}_{2}=\sqrt{2} / 2$. Thus,

$$
x=x\left(\hat{t}_{1}\right)=\hat{x}_{1}=\frac{\sqrt{3}}{2}, \quad x=x\left(\hat{t}_{2}\right)=\hat{x}_{2}=\frac{\sqrt{2}}{2} .
$$

However, it can be seen that

$$
\frac{-1-2 \hat{t}_{1}^{2}+4 \hat{t}_{1} \hat{x}_{1}}{1-6 \hat{t}_{1}^{2}+4 \hat{t}_{1} \hat{x}_{1}}=-1<0,
$$

which means that the inequality (2.21) is not satisfied for $t=\hat{t}_{1}$ and $x=\hat{x}_{1}$.

Note that the case $\hat{t}_{2}=\sqrt{2} / 2$ and $\hat{x}_{2}=\sqrt{2} / 2$ was considered in A1.1.

Therefore, $G$ does not have critical point in the interior of $\Omega$ in the case of (2.22).

II. Suppose that (2.23) is satisfied. The equation (2.23) as a quadratic one of $x$ with $\Delta:=17-44 t^{2}+36 t^{4}>0, t \in(0,1)$, has two roots, namely,

$$
x_{i}=x_{i}(t)=\frac{5+2 t^{2}+(-1)^{i+1} \sqrt{17-44 t^{2}+36 t^{4}}}{8 t}, \quad i=1,2
$$

a. Let $x=x_{1}$. Then, $\Phi\left(t, x_{1}(t)\right)=0$ is equivalent to the equation

$$
\begin{gathered}
\left(-39+154 t^{2}-208 t^{4}+96 t^{6}\right) \sqrt{17-44 t^{2}+36 t^{4}} \\
=161-844 t^{2}+1716 t^{4}-1600 t^{6}+576 t^{8}
\end{gathered}
$$

Squaring both sides of (2.25) leads to

$$
64 \gamma_{1}^{2}(t) \gamma_{2}^{4}(t)=0
$$

where for $t \in(0,1)$,

$$
\gamma_{1}(t):=-1+t^{2}, \quad \gamma_{2}(t):=-1+2 t^{2} .
$$


We see that there is a unique root $t=\sqrt{2} / 2$ of the equation (2.26), which does not satisfy (2.25). b. Let $x=x_{2}$. Then $\Phi\left(t, x_{2}(t)\right)=0$ is equivalent to the equation

$$
\begin{gathered}
-\left(-39+154 t^{2}-208 t^{4}+96 t^{6}\right) \sqrt{17-44 t^{2}+36 t^{4}} \\
=161-844 t^{2}+1716 t^{4}-1600 t^{6}+576 t^{8} .
\end{gathered}
$$

Squaring the both sides of (2.27) yields again the equation (2.26) having a unique root $t=\sqrt{2} / 2$, which satisfies (2.27) also. Since by (2.24), $x_{2}\left(t_{2}\right)=\sqrt{2} / 2$, we see that this case was considered in A1.1.

A2. It remains to consider $G$ in the boundary of $\Omega$.

(1) On the side $t=0$,

$$
G(0, x) \equiv-9>\Theta, \quad x \in[-1,1]
$$

(2) On the side $t=1$,

$$
G(1, x)=11-20 x+8 x^{2} \geq G(1,1)=-1>\Theta, \quad x \in[-1,1] .
$$

(3) On the side $x=-1$,

$$
G(t,-1)=-9-12 t+12 t^{2}+32 t^{3}+16 t^{4}=: \varrho_{1}(t), \quad t \in[0,1] .
$$

Since $\varrho_{1}^{\prime}(t)=0$ occurs only when

$$
t=t^{\prime}:=\frac{1}{4} \sqrt[3]{4+2 \sqrt{2}}+\frac{1}{2 \sqrt[3]{4+2 \sqrt{2}}}-\frac{1}{2}=0.23784 \cdots \in[0,1]
$$

and $\varrho_{1}^{\prime \prime}\left(t^{\prime}\right)>0$, we have

$$
\varrho_{1}(t) \geq \varrho_{1}\left(t^{\prime}\right)=\Theta, \quad t \in[0,1]
$$

(4) On the side $x=1$, we have

$$
G(t, 1)=-9+12 t+12 t^{2}-32 t^{3}+16 t^{4}:=\varrho_{2}(t), \quad t \in[0,1] .
$$

Since $\varrho_{2}$ is increasing,

$$
\varrho_{2}(t) \geq \varrho_{2}(0)=-9>\Theta, \quad t \in[0,1] .
$$

B. Suppose that $\zeta_{1}=0$. Then,

$$
\Psi_{1}=-1-4\left|\zeta_{2}\right|^{2}, \quad \Psi_{2}=4 \operatorname{Re} \zeta_{2}
$$


and, therefore,

$$
\operatorname{det} T_{3,1}(f)=-1+4 \operatorname{Re} \zeta_{2}-4\left|\zeta_{2}\right|^{2} \geq-9
$$

C. Suppose that $\zeta_{2}=0$ and $\zeta_{1}=r \mathrm{e}^{\mathrm{i} \theta}$, where $r \in(0,1]$ and $\theta \in[0,2 \pi)$. Then,

$$
\Psi_{1}=-1+4\left|\zeta_{1}\right|^{2}+12\left|\zeta_{1}\right|^{4}=-1+4 r^{2}+12 r^{4}
$$

and

$$
\Psi_{2}=4 \operatorname{Re} \zeta_{1}+4 \operatorname{Re}\left(\zeta_{1}^{2}\right)-24\left|\zeta_{1}\right|^{2} \operatorname{Re} \zeta_{1}=4 r \cos \theta+4 r^{2} \cos 2 \theta-24 r^{3} \cos \theta
$$

Thus,

$$
\operatorname{det} T_{3,1}(f)=G(r, \cos \theta), \quad r \in(0,1], \theta \in[0,2 \pi),
$$

where

$$
G(t, x):=-1+12 t^{4}+4 t x-24 t^{3} x+8 t^{2} x^{2}
$$

for $t \in(0,1]$ and $x \in[-1,1]$. Set

$$
x_{w}:=\frac{-1+6 t^{2}}{4 t}, \quad t \in(0,1] .
$$

Note that $-1<x_{w}$ holds for $t \in((-2+\sqrt{10}) / 6,1)$, and $x_{w}<1$ holds for $t \in$ $(0,(2+\sqrt{10}) / 6)$. Hence for $t \in((-2+\sqrt{10}) / 6,(2+\sqrt{10}) / 6)$, we have

$$
G(t, x) \geq G\left(t, x_{w}\right)=-\frac{3}{2}\left(2 t^{2}-1\right)^{2} \geq-\frac{1}{27}(22+4 \sqrt{10})=-1.283300 \cdots
$$

When $t<(-2+\sqrt{10}) / 6$, then

$$
G(t, x) \geq G(t,-1)=-1-4 t+8 t^{2}+24 t^{3}+12 t^{4}=: \phi_{1}(t) .
$$

Since $\phi_{1}^{\prime}(t)=0$ occurs only when $t=(-3+\sqrt{15}) / 6=0.145497 \cdots$ and

$$
\phi_{1}^{\prime \prime}((-3+\sqrt{15}) / 6)>0
$$

it follows that

$$
\phi_{1}(t) \geq \phi_{1}\left(\frac{1}{6}(-3+\sqrt{15})\right)=-\frac{4}{3}, \quad t \in\left(0, \frac{1}{6}(-2+\sqrt{10})\right) .
$$


When $t>(\sqrt{10}+2) / 6$, then

$$
G(t, x) \geq G(t, 1)=-1+4 t+8 t^{2}-24 t^{3}+12 t^{4}=: \phi_{2}(t) .
$$

Since $\phi_{2}$ is decreasing, we have

$$
\phi_{2}(t) \geq-1, \quad t \in\left(\frac{1}{6}(2+\sqrt{10}), 1\right) .
$$

Summarizing, from Parts A-C, it follows that the inequality (2.6) holds.

It remains to show that the inequality (2.6) is sharp. It is observed from (2.7), (2.14), (2.15) and (2.16) that $\operatorname{det} T_{3,1}(f)=\Theta$ holds when the following conditions are satisfied:

$$
r=t^{\prime}, \quad \cos \theta=-1, \quad s=1, \quad \sin (\psi+\alpha)=-1,
$$

where $t^{\prime}$ is given by (2.28), and where $\alpha$ is determined by the condition (2.12) with $\kappa_{1}$ and $\kappa_{2}$ given by (2.13). Then $\theta=\pi, \kappa_{1}=0$ and $\kappa_{2}=1 / 8\left((4+2 \sqrt{2})^{(2 / 3)}+(2\right.$ $\left.-\sqrt{2})(4+2 \sqrt{2})^{(1 / 3)}+8\right)=1.588825 \ldots$ Thus, $(2.12)$ is satisfied if we take $\alpha=\pi / 2$. Thus if we put $\psi=\pi$, then $\psi$ satisfies the fourth condition in (2.29). Now, let us consider a function $\tilde{p}$ which has the form (1.10) with $\zeta_{1}=-t^{\prime}$ and $\zeta_{2}=-1$, i.e.,

$$
\tilde{p}(z)=\frac{1-z^{2}}{1+2 t^{\prime} z+z^{2}}, \quad z \in \mathbb{D} .
$$

Since $\zeta_{1} \in \mathbb{D}$ and $\zeta_{2} \in \mathbb{T}$, from Lemma 1.2 it follows that $\tilde{p}$ belongs to $\mathcal{P}$. Therefore, the extremal function $f$ in the class $\mathcal{F}_{1}$ for which equality in (2.6) holds satisfies (2.1) with $p=\tilde{p}$.

\section{The Class $\mathcal{F}_{2}$}

Let $f \in \mathcal{F}_{2}$ be the form (1.1). Then by (1.4), there exists $p \in \mathcal{P}$ of the form (1.6) such that

$$
(1-z) \frac{f(z)}{z}=p(z), \quad z \in \mathbb{D} \text {. }
$$

Putting the series (1.1) and (1.6) into (3.1) by equating the coefficients, we get

$$
a_{2}=1+c_{1} \text { and } a_{3}=1+c_{1}+c_{2} \text {. }
$$

By (1.7), it follows that $A_{2}\left(\mathcal{F}_{2}\right)=3$ with the extremal function

$$
f(z)=\frac{z(1+z)}{(1-z)^{2}}, \quad z \in \mathbb{D} .
$$


Note that $a_{2}=0$ for the function $f \in \mathcal{F}_{2}$ given by

$$
f(z)=\frac{z}{1-z^{2}}, \quad z \in \mathbb{D} .
$$

Thus by Theorem 1.1, we have

Theorem 3.1 If $f \in \mathcal{F}_{2}$, then

$$
-8 \leq \operatorname{det} T_{2,1}(f) \leq 1 \text {. }
$$

Both inequalities are sharp.

Now, we estimate $\operatorname{det} T_{3,1}(f)$ in the class $\mathcal{F}_{2}$.

Theorem 3.2 If $f \in \mathcal{F}_{2}$, then

$$
\operatorname{det} T_{3,1}(f) \leq 48 \text {. }
$$

The inequality is sharp.

Proof By (3.2) and (1.7), we see that $\left|a_{2}\right| \leq 3$ and $\left|a_{3}\right| \leq 5$. As in the proof of Theorem 2.2, the inequality (2.5) holds with the function

$$
F(x, y):=2 x^{2} y-2 x^{2}-y^{2}+1, \quad(x, y) \in[0,3] \times[0,5] .
$$

Repeating argumentation in the in the proof of Theorem 2.2, we see that the function $F$ does not have any relative maxima in $(0,3) \times(0,5)$.

We consider $F$ on the boundary of $[0,3] \times[0,5]$.

(1) On the side $x=0$,

$$
F(0, y)=1-y^{2} \leq 1, \quad y \in[0,5]
$$

(2) On the side $x=3$,

$$
F(3, y)=-17+18 y-y^{2} \leq F(3,5)=48, \quad y \in[0,5] .
$$

(3) On the side $y=0$,

$$
F(x, 0)=1-2 x^{2} \leq 1, \quad x \in[0,3]
$$

(4) On the side $y=5$,

$$
F(x, 5)=-24+8 x^{2} \leq F(3,5)=48, \quad x \in[0,3] .
$$

Therefore, the inequality $F(x, y) \leq 48$ holds for all $(x, y) \in[0,3] \times[0,5]$, which in view of (2.5) shows (3.4).

For the function (3.3), $a_{2}=3$ and $a_{3}=5$, which makes the equality in (3.4). 
Theorem 3.3 If $f \in \mathcal{F}_{2}$, then

$$
\operatorname{det} T_{3,1}(f) \geq-\frac{4}{7}(5+4 \sqrt{2})=-6.0896309 \cdots .
$$

Proof Substituting (1.8) and (1.9) into (3.2) yields

$$
a_{2}=1+2 \zeta_{1}, \quad a_{3}=1+2 \zeta_{1}+2 \zeta_{1}^{2}+2\left(1-\left|\zeta_{1}\right|^{2}\right) \zeta_{2},
$$

for some $\zeta_{i} \in \overline{\mathbb{D}}(i=1,2)$. Therefore from (1.5), we get

$$
\operatorname{det} T_{3,1}(f)=\Psi_{1}+\Psi_{2},
$$

where

$$
\Psi_{1}:=4\left|\zeta_{1}\right|^{2}+12\left|\zeta_{1}\right|^{4}-4\left(1-\left|\zeta_{1}\right|^{2}\right)^{2}\left|\zeta_{2}\right|^{2}
$$

and

$$
\begin{aligned}
\Psi_{2}:= & 8 \operatorname{Re}\left(\zeta_{1}^{2}\right)+24\left|\zeta_{1}\right|^{2} \operatorname{Re} \zeta_{1}+8\left(1-\left|\zeta_{1}\right|^{2}\right) \operatorname{Re}\left(\zeta_{1} \bar{\zeta}_{2}\right) \\
& +8\left(1-\left|\zeta_{1}\right|^{2}\right) \operatorname{Re}\left(\zeta_{1}^{2} \bar{\zeta}_{2}\right)
\end{aligned}
$$

A. Suppose that $\zeta_{1} \zeta_{2} \neq 0$. Thus, $\zeta_{1}=r \mathrm{e}^{\mathrm{i} \theta}$ and $\zeta_{2}=s \mathrm{e}^{\mathrm{i} \psi}$ with $r, s \in(0,1]$ and $\theta, \psi \in[0,2 \pi)$. Then

$$
\Psi_{1}=4 r^{2}+12 r^{4}-4\left(1-r^{2}\right)^{2} s^{2} \geq-4+12 r^{2}+8 r^{4},
$$

and

$$
\Psi_{2}=8 r^{2} \cos 2 \theta+24 r^{3} \cos \theta+8\left(1-r^{2}\right) r s \sqrt{\kappa_{1}^{2}+\kappa_{2}^{2}} \sin (\psi+\alpha)
$$

where $\alpha$ is the quantity satisfying (2.12) with

$$
\kappa_{1}:=\sin \theta+r \sin 2 \theta, \quad \kappa_{2}:=\cos \theta+r \cos 2 \theta .
$$

Since $\sin (\psi+\alpha) \geq-1$ and $s \leq 1$, from (3.8) we have

$$
\Psi_{2} \geq 8 r^{2} \cos 2 \theta+24 r^{3} \cos \theta-8\left(1-r^{2}\right) r \sqrt{1+2 r \cos \theta+r^{2}} .
$$

Thus, from (3.6), (3.7) and (3.10), it follows that

$$
\operatorname{det} T_{3,1}(f) \geq-4 G(r, \cos \theta), \quad r \in(0,1], \theta \in[0,2 \pi),
$$

where, for $t \in[0,1]$ and $x \in[-1,1]$,

$$
G(t, x):=1-t^{2}-2 t^{4}-4 t^{2} x^{2}-6 t^{3} x+2\left(1-t^{2}\right) t \sqrt{g(t, x)}
$$


with

$$
g(t, x):=1+2 t x+t^{2} .
$$

Let $\Omega:=[0,1] \times[-1,1]$ and

$$
\Theta:=\frac{1}{7}(5+4 \sqrt{2})=1.522407 \cdots .
$$

Now, we will show that

$$
\max \{G(t, x):(t, x) \in \Omega\}=\Theta .
$$

A1. For this, we first we find the critical points of $G$ in the interior of $\Omega$, i.e., in $(0,1) \times(-1,1)$. Note first that $g(t, x)>0$ in $(0,1) \times(-1,1)$. From the equation

$$
\frac{\partial G}{\partial x}(t, x)=-8 t^{2} x-6 t^{3}+2\left(1-t^{2}\right) t^{2} g(t, x)^{-1 / 2}=0,
$$

it follows that

$$
0<g(t, x)^{-1 / 2}=\frac{4 x+3 t}{1-t^{2}}
$$

Hence,

$$
4 x+3 t>0 \text {. }
$$

Under the condition (3.14), the equation (3.13) by substituting (3.12) can be equivalently written as

$$
32 t x^{3}+16\left(4 t^{2}+1\right) x^{2}+2\left(21 t^{3}+12 t\right) x+8 t^{4}+11 t^{2}-1=0 .
$$

Differentiating $G$ with respect to $t$ leads to the equation

$$
\begin{aligned}
\frac{\partial G}{\partial t}(t, x)= & -2 t-8 t^{3}-8 t x^{2}-18 t^{2} x+2\left(1-3 t^{2}\right) g(t, x)^{1 / 2} \\
& +2\left(1-t^{2}\right)(x+t) \operatorname{tg}(t, x)^{-1 / 2}=0,
\end{aligned}
$$

which by using (3.13) is equivalent to

$$
8 t^{2} x^{2}+\left(10 t^{3}+4 t\right) x+7 t^{2}-1=0 .
$$

The above equation is a quadratic one of $x$ with $\Delta=\Delta(t):=12-36 t^{2}+25 t^{4}$, and $\Delta(t) \geq 0$ if and only if $t \in\left(0, t_{1}\right] \cup\left[t_{2}, 1\right)$, where

$$
t_{1}:=\frac{1}{5} \sqrt{2(9-\sqrt{6})}=0.723906 \ldots, \quad t_{2}:=\frac{1}{5} \sqrt{2(9+\sqrt{6})}=0.957057 \ldots
$$


For $\Delta\left(t_{1}\right)=0$, the equation (3.16) has a unique root

$$
x_{0}^{\prime}:=-\frac{2+5 t_{1}^{2}}{8 t_{1}}=\frac{-14+\sqrt{6}}{4 \sqrt{18-2 \sqrt{6}}}=-0.797790 \cdots .
$$

Analogously, for $\Delta\left(t_{2}\right)=0$, the equation (3.16) has a unique root

$$
x_{0}^{\prime \prime}:=-\frac{2+5 t_{2}^{2}}{8 t_{2}}=-\frac{14+\sqrt{6}}{4 \sqrt{18+2 \sqrt{6}}}=-0.859366 \cdots .
$$

It can be verified that the equation in (3.15) does not hold for $t=t_{1}$ and $x=x_{0}^{\prime}$, and for $t=t_{2}$ and $x=x_{0}^{\prime \prime}$.

Assume now that $t \in\left(0, t_{1}\right) \cup\left(t_{2}, 1\right)$. Thus, there are two roots $x_{1}$ and $x_{2}$ of (3.16), namely

$$
x_{j}=x_{j}(t):=\frac{-2-5 t^{2}+(-1)^{j} \sqrt{12-36 t^{2}+25 t^{4}}}{8 t}, \quad j=1,2 .
$$

I. Consider the case $x=x_{1}$. Note that $x_{1}>-1$ is equivalent to

$$
-2+8 t-5 t^{2}>\sqrt{12-36 t^{2}+25 t^{4}}
$$

Observe that $-2+8 t-5 t^{2}>0$ if and only if $t \in\left(t_{3}, 1\right)$, where

$$
t_{3}:=\frac{1}{5}(4-\sqrt{6})=0.310102 \cdots
$$

Thus for $t \in\left(t_{3}, t_{1}\right) \cup\left(t_{2}, 1\right)$ by squaring the both sides of (3.17), we get the inequality

$$
80 t^{3}-120 t^{2}+32 t+8<0
$$

which holds if and only if $t \in\left(t_{4}, t_{1}\right) \cup\left(t_{2}, 1\right)=: J_{1}$, where $t_{4}:=(5+\sqrt{65}) / 20=$ $0.653112 \cdots$. Moreover, $x_{1}<1$ is equivalent to the inequality

$$
-2-8 t-5 t^{2}<\sqrt{12-36 t^{2}+25 t^{4}}
$$

which is true for $t \in J_{1}$.

Substituting $x=x_{1}$ into the equation (3.15), we get

$$
Q_{1}(t) \sqrt{12-36 t^{2}+25 t^{4}}=Q_{2}(t)
$$

where for $t \in J_{1}$,

$$
Q_{1}(t):=1-4 t^{2}+3 t^{4}, \quad Q_{2}(t):=-2+7 t^{2}-6 t^{4}+t^{6} .
$$


Since $Q_{1}(t)<0$ for $t \in J_{1}$, and $Q_{2}(t)<0$ for $t \in\left(t_{4}, t_{5}\right)$, where $t_{5}:=$ $\sqrt{10-2 \sqrt{17}} / 2=0.662153 \ldots$, by squaring both sides of (3.19) we equivalently get the equation

$$
8\left(1-2 t^{2}\right)\left(1-8 t^{2}+14 t^{4}\right)(1-t)^{3}(1+t)^{3}=0
$$

which has no solution for $t \in\left(t_{4}, t_{5}\right)$.

II. Consider now the case $x=x_{2}$. Note that $x_{2}>-1$ is equivalent to

$$
2-8 t+5 t^{2}<\sqrt{12-36 t^{2}+25 t^{4}}
$$

Since $2-8 t+5 t^{2}<0$ for $t \in\left(t_{3}, 1\right)$, where $t_{3}$ is given by (3.18), consider $t \in\left(0, t_{3}\right]$. By squaring both sides of (3.21), we get the inequality

$$
80 t^{3}-120 t^{2}+32 t+8 \geq 0
$$

which is true for $t \in\left(0, t_{3}\right]$. Thus, $x_{2}>-1$ holds for all $t \in\left(0, t_{1}\right) \cup\left(t_{2}, 1\right)$. Moreover, $x_{2}<1$ is equivalent to the inequality

$$
2+8 t+5 t^{2}>\sqrt{12-36 t^{2}+25 t^{4}}
$$

By squaring both sides, we equivalently get the inequality

$$
80 t^{3}+120 t^{2}+32 t-8>0
$$

which is true for $t \in\left(t_{6}, t_{1}\right) \cup\left(t_{2}, 1\right)=: J_{2}$, where $t_{6}:=(-5+\sqrt{65}) / 20=$ $0.153113 \cdots$. Thus, we restrict our consideration for $t \in J_{2}$.

Substituting $x=x_{2}$ into the equation (3.15) yields the equation (3.20), which has three roots in $J_{2}$, namely,

$$
\begin{aligned}
& \tilde{t}_{1}=\frac{\sqrt{2}}{2}=0.707106 \cdots, \quad \tilde{t}_{2}=\frac{1}{14} \sqrt{56+14 \sqrt{2}}=0.621875 \cdots, \\
& \tilde{t}_{3}=\frac{1}{14} \sqrt{56-14 \sqrt{2}}=0.429766 \ldots
\end{aligned}
$$

Since $x_{2}\left(\tilde{t}_{1}\right)=\tilde{x}_{1}=-\sqrt{2} / 2$, it follows that $4 \tilde{x}_{1}+3 \tilde{t}_{1}<0$ which contradicts $(3.14)$. Therefore, $\left(\tilde{t}_{1}, \tilde{x}_{1}\right)$ is not a critical point of $G$.

Since

$$
x_{2}\left(\tilde{t}_{2}\right)=\tilde{x}_{2}=\frac{7(\sqrt{2}-4)}{4 \sqrt{56+14 \sqrt{2}}}=-0.5197553794 \cdots,
$$

it follows that

$$
4 \tilde{x}_{2}+3 \tilde{t}_{2}=\frac{-16+10 \sqrt{2}}{\sqrt{56+14 \sqrt{2}}}=-0.213394046 \cdots<0
$$


which contradicts (3.14). Therefore, $\left(\tilde{t}_{2}, \tilde{x}_{2}\right)$ is not a critical point of $G$.

Further,

$$
x_{2}\left(\tilde{t}_{3}\right)=\tilde{x}_{3}=\frac{-5+3 \sqrt{2}}{\sqrt{56-14 \sqrt{2}}}=-0.1258756 \cdots
$$

Since

$$
4 \tilde{x}_{3}+3 \tilde{t}_{3}=\frac{-8+9 \sqrt{2}}{\sqrt{56-14 \sqrt{2}}}=0.7857962 \cdots>0
$$

then $\left(\tilde{t}_{3}, \tilde{x}_{3}\right)$ satisfies (3.14), and therefore it is a unique critical point of $G$. Denote

$$
\begin{aligned}
\lambda_{1}:=\frac{\partial^{2} G}{\partial t^{2}}\left(\tilde{t}_{3}, \tilde{x}_{3}\right) & =\frac{1}{14}(8-93 \sqrt{2})=-8.822990 \cdots, \\
\lambda_{2} & :=\frac{\partial^{2} G}{\partial t \partial x}\left(\tilde{t}_{3}, \tilde{x}_{3}\right)=\frac{1}{7}(-43+23 \sqrt{2}), \\
\lambda_{3} & :=\frac{\partial^{2} G}{\partial x^{2}}\left(\tilde{t}_{3}, \tilde{x}_{3}\right)=\frac{1}{7}(44-39 \sqrt{2}) .
\end{aligned}
$$

Since $\lambda_{1}<0$ and

$$
\lambda_{1} \lambda_{3}-\lambda_{2}^{2}=\frac{1}{7}(128-32 \sqrt{2})=11.8207380 \cdots>0,
$$

the function $G$ has a local maximum at $\left(\tilde{t}_{3}, \tilde{x}_{3}\right)$.

A2. It remains to consider $G$ in the boundary of $\Omega$.

(1) On the side $t=0$,

$$
G(0, x) \equiv 1<\Theta, \quad x \in[-1,1] .
$$

(2) On the side $t=1$,

$$
G(1, x)=-2-6 x-4 x^{2} \leq G\left(1,-\frac{3}{4}\right)=\frac{1}{4}<\Theta, \quad x \in[-1,1]
$$

(3) On the side $x=-1$,

$$
G(t,-1)=1+2 t-7 t^{2}+4 t^{3}=: \varrho_{1}(t), \quad t \in[0,1] .
$$

Since $\varrho_{1}^{\prime}(t)=0$ if and only if $t=1 / 6$ or $t=1$, we see that

$$
\varrho_{1}(t) \leq \varrho_{1}\left(\frac{1}{6}\right)=\frac{125}{108}<\Theta, \quad t \in[0,1]
$$


(4) On the side $x=1$, we have

$$
G(t, 1)=1+2 t-3 t^{2}-8 t^{3}-4 t^{4}=: \varrho_{2}(t), \quad t \in[0,1]
$$

Since $\varrho_{2}^{\prime}(t)=0$ occurs only when $t=(\sqrt{3}-1) / 4 \in(0,1)$ and $\varrho_{2}^{\prime \prime}((\sqrt{3}-1) / 4)=$ $-6(1+\sqrt{3})<0$, it follows that

$$
\varrho_{2}(t) \leq \varrho_{2}\left(\frac{1}{4}(\sqrt{3}-1)\right)=\frac{3}{16}(3+2 \sqrt{3})=1.2120 \cdots<\Theta, \quad t \in[0,1] .
$$

B. Suppose that $\zeta_{1}=0$. Then,

$$
\operatorname{det} T_{3,1}(f)=-4\left|\zeta_{2}\right|^{2} \geq-4
$$

C. Suppose that $\zeta_{2}=0$ and $\zeta_{1}=r \mathrm{e}^{\mathrm{i} \theta}$, where $r \in(0,1]$ and $\theta \in[0,2 \pi)$. Then,

$$
\Psi_{1}=4\left|\zeta_{1}\right|^{2}+12\left|\zeta_{1}\right|^{4}=4 r^{2}+12 r^{4}
$$

and

$$
\Psi_{2}=8 \operatorname{Re}\left(\zeta_{1}^{2}\right)+24\left|\zeta_{1}\right|^{2} \operatorname{Re} \zeta_{1}=8 r^{2} \cos 2 \theta+24 r^{3} \cos \theta
$$

Thus,

$$
\operatorname{det} T_{3,1}(f)=G(r, \cos \theta), \quad r \in(0,1], \theta \in[0,2 \pi),
$$

where

$$
G(t, x)=-4 t^{2}+12 t^{4}+24 t^{3} x+16 t^{2} x^{2}
$$

for $t \in(0,1]$ and $x \in[-1,1]$. Set

$$
x_{w}:=-\frac{3}{4} t, \quad t \in(0,1) .
$$

Since $-1<x_{w}<0$,

$$
G(t, x) \geq G\left(t, x_{w}\right)=-4 t^{2}+3 t^{4}:=\phi(t) .
$$

By the fact that $\phi^{\prime}(t)=0$ occurs when $t=\sqrt{6} / 3=0.816496 \cdots$ and $\phi^{\prime \prime}(\sqrt{6} / 3)=$ $16>0$, we see that

$$
\phi(t) \geq \phi\left(\frac{\sqrt{6}}{3}\right)=-\frac{4}{3}, \quad t \in(0,1) .
$$

Summarizing, from Parts A-C, it follows that the inequality (3.5) holds. 
Now, we discuss the sharpness of (3.5). It is observed from (3.7), (3.10) and (3.11) that det $T_{3,1}(f)=-4 \Theta$ holds when the following conditions are satisfied:

$$
r=\tilde{t}_{3}, \quad \cos \theta=\tilde{x}_{3}, \quad s=1, \quad \sin (\psi+\alpha)=-1,
$$

where $\tilde{t}_{3}$ and $\tilde{x}_{3}$ are given by (3.22) and (3.23), respectively, and $\alpha$ is determined by the condition (2.12) with $\kappa_{1}$ and $\kappa_{2}$ given by (3.9). Set $\theta=\operatorname{Arccos}\left(\tilde{x}_{3}\right)$ so that it satisfies the second condition in (3.24). Then, $\kappa_{1}=0.884712 \cdots>0$ and $\kappa_{2}=$ $-0.542022 \cdots<0$. Thus, $(2.12)$ is satisfied if we take

$$
\alpha=-\operatorname{Arccos}\left(\frac{\kappa_{1}}{\sqrt{\kappa_{1}^{2}+\kappa_{2}^{2}}}\right)=-0.549672 \cdots
$$

Thus if we put

$$
\psi=\frac{3 \pi}{2}-\alpha=5.262061 \cdots
$$

then $\psi$ satisfies the fourth condition in (3.24). Now, let us consider a function $\tilde{p}$ which has the form (1.10) with $\zeta_{1}=\tilde{t}_{3} \mathrm{e}^{\mathrm{i} \theta}$ and $\zeta_{2}=\mathrm{e}^{\mathrm{i} \psi}$. Since $\zeta_{1} \in \mathbb{D}$ and $\zeta_{2} \in \mathbb{T}$, in view of Lemma 1.2, we see that $\tilde{p}$ belongs to the class $\mathcal{P}$. Taking now a function

$$
\tilde{f}(z)=\frac{z \tilde{p}(z)}{(1-z)}, \quad z \in \mathbb{D}
$$

we see that $\tilde{f} \in \mathcal{F}_{2}$ and $\operatorname{det} T_{3,1}(\tilde{f})=-4 \Theta$, which completes the proof.

Availability of data and material Not applicable. The manuscript has no associated data.

\section{Declarations}

Conflicts of interest The authors declare that they have no conflict of interest.

Open Access This article is licensed under a Creative Commons Attribution 4.0 International License, which permits use, sharing, adaptation, distribution and reproduction in any medium or format, as long as you give appropriate credit to the original author(s) and the source, provide a link to the Creative Commons licence, and indicate if changes were made. The images or other third party material in this article are included in the article's Creative Commons licence, unless indicated otherwise in a credit line to the material. If material is not included in the article's Creative Commons licence and your intended use is not permitted by statutory regulation or exceeds the permitted use, you will need to obtain permission directly from the copyright holder. To view a copy of this licence, visit http://creativecommons.org/licenses/by/4.0/.

\section{References}

1. Ali, F., Thomas, D. K., Vasudevarao, A.: Toeplitz determinants whose elements are the coefficients of analytic and univalent functions. Bull. Aust. Math. Soc. 97(2), 253-264 (2018) 
2. Biernacki, M.: Sur la représentation conforme des domaines linéairement accessibles. Prace Mat.-Fiz. 44, 293-314 (1936)

3. Carathéodory, C.: Über den Variabilitatsbereich der Koeffizienten von Potenzreihen, die gegebene werte nicht annehmen. Math. Ann. 64, 95-115 (1907)

4. Causey, W.M., Merkes, E.P.: Radii of starlikeness of certain classes of analytic functions. J. Math. Anal. Appl. 31, 579-586 (1970)

5. Cho, N.E., Kowalczyk, B., Kwon, O.S., Lecko, A., Sim, Y.J.: Some coefficient inequalities related to the Hankel determinant for strongly starlike functions of order alpha. J. Math. Ineq. 11(2), 429-439 (2017)

6. Cho, N.E., Kowalczyk, B., Kwon, O.S., Lecko, A., Sim, Y.J.: The bounds of some determinants for starlike functions of order alpha. Bull. Malays. Math. Sci. Soc. 41(1), 523-535 (2018)

7. Cho, N.E., Kowalczyk, B., Lecko, A.: Sharp bounds of some coefficient functionals over the class of functions convex in the direction of the imaginary axis. Bull. Aust. Math. Soc. 100, 86-96 (2019)

8. Cudna, K., Kwon, O.S., Lecko, A., Sim, Y.J., Smiarowska, B.: The second and third-order Hermitian Toeplitz determinants for starlike and convex functions of order $\alpha$. Bol. Soc. Mat. Mexicana 26, 361375 (2020)

9. Goodman, A.W.: Univalent Functions. Mariner, Tampa, Florida (1983)

10. Jastrzȩbski, P., Kowalczyk, B., Kwon, O.S., Lecko, A., Sim, Y. J.: Hermitian Toeplitz determinants of the second and third-order for classes of close-to-star functions. Rev. R. Acad. Cienc. Exactas Fís. Nat. 114, Art. 166, 1-14 (2020)

11. Kaplan, W.: Close to convex schlicht functions. Mich. Math. J. 1, 169-185 (1952)

12. Kowalczyk, B., Kwon, O.S., Lecko, A., Sim, Y.J.: The bounds of some determinants for functions of bounded turning of order alpha. Bulletin de la Société Des Sciences et des Lettres de Łódź: Recherches sur les déformations LXVI I(1), 107-118 (2017)

13. Kowalczyk, B., Kwon, O.S., Lecko, A., Sim, Y.J., Śmiarowska, B.: The Third-Order Hermitian Toeplitz Determinant for Classes of Functions Convex in One Direction. Bull. Malays. Math. Sci. Soc. 43, 3143-3158 (2020)

14. Kowalczyk, B., Lecko, A., Sim, Y.J.: The sharp bound of the Hankel determinant of the third kind for convex functions. Bull. Aust. Math. Soc. 97, 435-445 (2018)

15. Kowalczyk, B., Lecko, A., Śmiarowska, B.: Sharp inequalities for Hermitian Toeplitz determinants for strongly starlike and strongly convex functions. J. Math. Ineq. 15(1), 323-332 (2021)

16. Kowalczyk, B., Lecko, A., Lecko, M., Sim, Y.J.: The sharp bound of the third Hankel determinant for some classes of analytic functions. Bull. Korean Math. Soc. 55(6), 1859-1868 (2018)

17. Kwon, O.S., Lecko, A., Sim, Y.J.: The bound of the Hankel determinant of the third kind for starlike functions. Bull. Malays. Math. Sci. Soc. 42, 767-780 (2019)

18. Lecko, A., Sim, Y.J., Smiarowska, B.: The sharp bound of the Hankel determinant of the third kind for starlike functions of order 1/2. Complex Anal. Oper. Theory 13, 2231-2238 (2019)

19. Lee, S.K., Ravichandran, V., Supramanian, S.: Bound for the second Hankel determinant of certain univalent functions. J. Inequal. Appl. 2013(281), 1-17 (2013)

20. Lewandowski, Z.: Sur l'identiteé de certaines classes de fonctions univalentes, I, II. Ann. Univ. Mariae Curie-Skłodowska Sect. A 12, 131-146 (1958); 14, 19-46 (1960)

21. MacGregor, T.H.: The radius of univalence of certain analytic functions. Proc. Am. Math. Soc. 14, 514-520 (1963)

22. Pommerenke, C.: Univalent Functions. Vandenhoeck \& Ruprecht, Göttingen (1975)

23. Ravichandran, V., Verma, S.: Generalized Zalcman conjecture for some classes of analytic functions. J. Math. Anal. Appl. 450(1), 592-605 (2017)

24. Reade, M.: On close-to-convex univalent functions. Mich. Math. J. 3, 59-62 (1955)

25. Robertson, M.S.: Analytic functions star-like in one direction. Am. J. Math. 58, 465-472 (1936)

26. Sakaguchi, K.: The radius of convexity for certain class of regular functions. J. Nara Gakugei Univ. Natur. Sci. 12, 5-8 (1964)

Publisher's Note Springer Nature remains neutral with regard to jurisdictional claims in published maps and institutional affiliations. 